\title{
High-throughput Quantitative STEM Mass Measurement in Statistically Robust Populations of Supported Metal Nanoparticles
}

\author{
Stephen D. House ${ }^{1}$, Yuxiang Chen ${ }^{2}$, Rongchao Jin ${ }^{2}$, Judith C. Yang ${ }^{1}$ \\ 1. Department of Chemical and Petroleum Engineering, University of Pittsburgh, Pittsburgh, PA (USA) \\ 2. Department of Chemistry, Carnegie Mellon University, Pittsburgh, PA (USA)
}

The rational design of nanoparticle-based material systems - such as for heterogeneous catalyst, optoelectronic, or medical applications - requires an understanding of the structure-property-performance relationship, which relies on the accurate knowledge of the structure of the synthesized materials at the relevant length scales. This is particularly important for particles below a few nanometers in size, small structural differences can produce significant changes in physical or chemical properties. Quantitative scanning transmission electron microscopy (STEM) provides a way overcome the inherent 2D projection nature of TEM micrographs and extract the 3D structural (or compositional) information from a single (or for certain approaches, more) micrograph. The first practical quantitative STEM technique for measuring the scattering cross-section of nanoparticles - was introduced two decades ago by Singhal et al. [1] for the (long-gone) VG HB501 STEM. The advent of aberration-corrected microscopes spurred renewed interest in quantitative STEM nearly a decade ago, leading to the development of atomicresolution quantitative STEM methodologies [2-4]. The potential offered by these approaches, for true atom-by-atom 3D reconstructions of a given nanostructure, is significant. However, they also share a few limitations, in particular the specialized expertise - in advanced image simulation or statistical likelihood analysis [5] - and expensive instruments required, which restricts the potential user base, and the time-consuming nature of the acquisition and analysis, which limits the number of nanoparticles that can be examined. The latter brings into question how representative the nanoparticle (or few) that were selected are of the sample as a whole, a critical issue for systems like catalysis where the relevant properties arise from an ensemble that may or may not be uniform.

To address these issues, we adapted and improved the quantitative STEM methodology originally proposed by Singhal et al. [1] for use on modern conventional (non-aberration-corrected) S/TEMs without the need for any hardware modifications or image simulation. This technique enables the size, mass (via the cross-section), and basic shape information of nanoparticles and can be used both as an alternative - for facilities without access to aberration-corrected instruments - or a complement to atomic-resolution quantitative STEM. The methods for performing the necessary instrument and image calibrations are discussed in this presentation along with a detailed investigation into how the accuracy and/or precision of the measurement is impacted by each parameter and the procedures for gauging it. A portion of one such calibration - determining detector collection angles and response efficiency - is shown in Figure 1. Atomically precise gold nanoparticles $\left(\mathrm{Au}_{25}(\mathrm{PET})_{18}\right.$ and $\left.\mathrm{Au}_{144}(\mathrm{PET})_{60}\right)$ were used to validate the technique, Figure 2, revealing an instrument-dependent uniform scaling factor of approximately 2 for the JEOL JEM2100F on which this work was performed. This discrepancy can be corrected for via a calibration standard, but its origin and how to remove it altogether is being explored further.

To facilitate the high-throughput necessary to analyze statistically robust populations of hundreds to thousands of particles we have also written user-friendly program to perform the quantitative analysis, cutting time requirements from days or weeks down to minutes. The program automatically optimizes 
the analysis parameters, applying them consistently across the dataset, enhancing the reproducibility and transferability of the results as well as rendering the statistics more meaningful. The program is available for download while its functionality continues to be improved and expanded. The large sample population analyzable with the automated program make this quantitative STEM technique well-suited for applications such as rapid screening and gauging the monodispersity of synthesized samples. [6].

\section{References:}

[1] A Singhal, et al., Ultramicroscopy 67 (1997), p. 191-206.

[2] JM LeBeau and S Stemmer, Ultramicroscopy 108 (2008), p. 1653-1658.

[3] A Rosenauer, et al., Ultramicroscopy 111 (2011), p. 1316-1327.

[4] L Jones, et al., Nano Letters 14 (2014), p. 6336-6341.

[5] A Van Aert, et al., Physical Review B 87 (2013), p.064107.

[6] JCY and SDH acknowledge the support for this work by DOE BES through grant DE FG0203ER15476 and NSF DMREF through contract No CHE-1534630. RJ and YC acknowledge the financial support from the Air Force Office of Scientific Research under AFOSR Award No. FA955015-1-9999 (FA9550-15-1-0154) and the Camille Dreyfus Teacher-Scholar Awards Program.
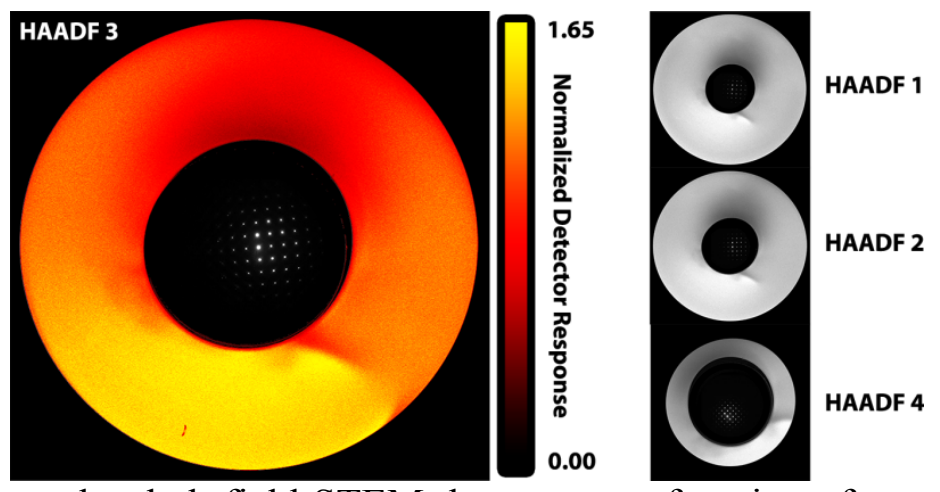

Figure 1. Maps of the annular dark-field STEM detector as a function of camera length. The detector was mapped in true angular space, reflecting the actual electron trajectories during imaging and allowing the detector efficiency and collection angles to be gathered from a single map. The corresponding STEM diffraction patterns, used for calibrating the angles, have been overlaid. The HAADF 3 map's response has been normalized against the mean.

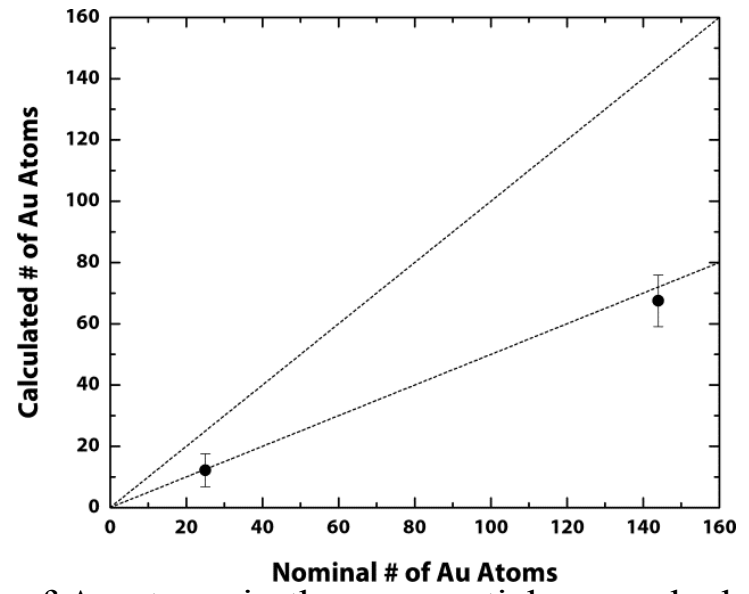

Figure 2. Plot of the number of $\mathrm{Au}$ atoms in the nanoparticles as calculated by quantitative STEM vs. the nominal count. The black circles are the population means, the error bars are the population standard deviations, and the dashed lines are visual guides with slopes of 1 and $1 / 2$. 